\title{
Community Psychiatry from Academia
}

\author{
B. J. Marthews, Department of Psychiatry, St George's Hospital Medical School, London SW17; and E. K. McLEAN, \\ Springfield Hospital, London SW17
}

The community approaches which can be used in psychiatric practice are not so often in evidence in academic departments of psychiatry. One of us (BJM), on commencing as a Clinical Research Fellow within the Department of Psychiatry, St George's Hospital Medical School, was keen to spend his clinical time (set aside for training purposes) in a community-orientated way.

After discussion, we formulated a plan to attempt to provide a long-term, community-based follow-up service to young psychotic patients and their families within $\mathrm{Dr}$ McLean's catchment area, the southern part of the Battersea district of the London Borough of Wandsworth. It is a densely populated inner city area, with large amounts of local authority housing and a large immigrant population. The level of psychotic illness in the area is high. In the first six months of 1983, there were 24 cases of schizophrenia and paranoid illness, and twelve of major affective disorders, out of a total of 86 admissions.

We recognized that, despite the existence of a well-developed community psychiatric nursing service, many young patients who had had psychotic illnesses fell out of the follow-up systems, deteriorated and re-presented, usually as acute admissions after a recurrence of psychosis. The aims of this deliberately naturalistic project were to assess why this group tend to fall out of follow-up and whether any improvement could be made in the system. In this group are many potential new long-stay patients, who will be a large drain on adult long-stay resources in the future. Improving the relapse rate has considerable implications for future resource planning.

Providing a more sure method of follow-up, in the home setting, would perhaps lead to an opportunity for patients and family to develop a relationship with a doctor in post for a relatively long period. We hoped that this system would enable a closer watch to be kept on medication compliance and relapse, perhaps avoiding re-admission. Setting the follow-up outside the institution was designed to help the process of engaging the patients' families and examining their feelings about the illness. In our view, staff on an admission ward naturally concentrate on the patient, the family may interpret this as rejection; the project was designed in part to attempt to bridge that gap.

Involving the family was important in the development of a more secure supportive framework for individual patients. There was no intention to involve these families in 'family therapy' as usually described, but more an attempt to develop links with them to avoid relapses. The educational aspects of this process were recognized as important.

It was decided not to remove these patients from the normal follow-up system of out-patient appointments and, where appropriate, community psychiatric nurse involvement. We decided initially to operate this approach for one year, and then review its operation. This paper is the result of that review.

Patients were selected jointly during the normal weekly ward round. All members of the firm knew of the project, $\mathrm{Dr}$ Matthews spent at most one session per week on it.

\section{Progress}

Out of an admission total of about 45 cases of schizophrenia, eight patients were collected in the index year. All were under the age of 35 (three men and five women), and all had been hospitalized with psychotic illnesses without marked affective features. Seven of the eight cases were presenting for the first time, the other case being of a man with a chronic schizophrenic illness with frequent relapses into acute psychosis. Three patients were of Afro-Caribbean origin. All of the cases had been initially treated as in-patients in Springfield Hospital, London. Treatment followed standard lines, initially with oral or intramuscular phenothiazines, followed by transfer to depot medication if agreeable. Other treatments were used as appropriate.

Three patients were discharged directly from the ward to their family homes, one patient had a new council flat to move into. The other four patients were referred on discharge from the ward to the day hospital to attend from their family homes. Three rapidly stopped attending, with one patient coming intermittently.

\section{Medication}

Three patients were prescribed depot medication, the others refusing to accept it. One of the depot patients was receiving treatment under Section 37 of the Mental Health Act (1983). Injections were administered by the community psychiatric nurse attached to the firm in his regular clinic.

All of the other patients were offered depot medication but refused to accept it. All patients were reluctant to take any medication at all and repeated persuasion was necessary. Two patients stopped taking oral medication prematurely and relapsed, but then resumed. One patient stopped immediately after discharge from hospital and relapsed gradually. One is at present reducing his medication against advice!

\section{Role of the home visits}

Regular contact with one known member of the firm was one of the primary reasons for setting up the project. Patients seemed to fall into two groups with regard to this. Three cases did not value the contact. This was shown by their repeated failure to be at home at arranged times, chronic difficulty in contacting the patients and their relatives, and finally, in two cases, the request that visits be discontinued.

In both of these last cases, the patients seemed to find it exceptionally hard to come to terms with their illness. Their attempt to deny its importance was demonstrated in their 
failure to take medication. We felt that the visits from a psychiatrist intruded into their denial such that they needed to end the contact. In both of these cases the patients were of Afro-Caribbean origin and intelligent, their hopes for the future had been severely compromised by their illness.

The third 'disinterested' case was an older ( 33 year old) white man on Section 37 of the Mental Health Act. Both he and his parents were chronically psychotic. Denial of illness was strong in the whole family.

One case has not been part of the project long enough to assess her attitude to it.

Four cases seemed to value the meetings. In three of them, this appreciation was shared equally by relatives and patients. In the other case, the patient seemed to fall into the 'denying' group, whilst her parents (who were heavily burdened by her) were desperate for help.

All of the families in this group were highly concerned about their relatives' illness, and their prospects. With the development of a relationship, some parents became able to reveal their feelings of guilt. Education about the factors in the causation of schizophrenic illnesses was effective in reducing some of this. Generally, families valued feeling involved more consistently, although this appreciation only came through after some time. At first the families seemed willing to accept the treatment offered in a fatalistic way, and were only able to offer their concerns and anxieties later on, as the relationship gelled. On questioning, relatives said they valued having contact with one doctor who they felt had come to know their situation well.

Despite continuing contact, compliance with medication was still very variable, and the educative aims of the meetings came into prominence. Clear, sensible explanation of the needs for, and effect of medication repeatedly proved to be the most frequent topic for conversation. In two cases, patients stopped medication against advice and quickly relapsed. However, the close contact and the therapeutic relationship made it possible for them to rapidly restart before symptoms became serious. In another case, this education function led to the reaching of an agreement which prevented an uncontrolled stopping of medication.

Generally, patients had little understanding of the reasons for taking medication and, conspicuously, no knowledge of its protective effect against relapse. Side effects were not a major problem, but explanation of their occurrence was reassuring.

Another aspect of the educational process was discussion of the causation and implications of their illness. The group demonstrating denial tended to avoid such topics, but the other group welcomed their introduction. They formed a useful basis for discussion about career prospects and the reasons for avoiding external stress and examinations. All of these patients found it extremely hard to find employment after leaving hospital, with only one being in employment at the present time. Advice and support were given as appropriate.

Supervision of cases proved to be quite difficult, close liaison with the ward-based team was important. As mentioned, two patients ended contact, and have not been re-referred. In one case, gradual deterioration was monitored, and finally a voluntary re-admission to hospital negotiated. The patient's prior admission, with similar symptoms, had been compulsory following a suicide attempt. Other patients have been seen at intervals varying from one week to two months. Visits last between 30 minutes to one hour. Relatives are generally present, and are able to give their view of the situation, which is sometimes at variance with the patient's!

\section{Conclusions}

This system facilitated the development of relationships with patients and their relatives from a group with a generally poor prognosis. Acute relapse in three patients may have been prevented. Time spent with relatives has led to their becoming a more constructive part of the network aiding patients' recovery.

However, in some cases, this system did not help and the combined resistance of patients and families was not overcome.

The role described is clearly not restricted to a psychiatrist. However, the authoritative figure of the 'doctor' was able to contribute with added weight. Much of the work was educative, and facts and concepts had to be put over in a way comprehended by lay people. A clear understanding of the illness and treatment concepts is therefore needed. The role could be fulfilled by a CPN or psychiatrically trained social worker, although we feel that at present it fills a slightly different niche from that usually occupied by these professionals.

We intend to review these cases after a further year.

ACKNOWLEDGEMENTS

We would like to thank the Wolfson Charitable Trust who is funding Dr Matthews as Clinical Research Fellow, and Mrs Jill Edwards who typed the manuscript.

\section{'Statistical Bulletin', DHSS}

The DHSS Statistical Bulletin (Bulletin 1/85) on 'Mental Illness Hospitals and Units in England' is now available. This report presents the results of the 1983 Mental Health Enquiry for mental illness hospitals and units and examines the trends over the period from 1973. The statistics analysed are mainly of admission to, and discharges from, mental illness hospitals and units. Numbers of resident patients in these hospitals and units are also given. The description of the main trends over the period from 1973 to 1983 is followed by a more detailed analysis of the 1983 figures compared with those in the immediately preceding years. Similar statistics for mental handicap hospitals and units are presented in a separate Statistical Bulletin (Bulletin 2/85).

A copy of the Statistical Bulletin is available for reference in the College Library, and further copies may be obtained from: Information Division, DHSS, Cannons Park, Government Buildings, Honeypot Lane, Stanmore HA7 1AY. 